\title{
RELACION DE LOS NIVELES DE AUTOEFICACIA Y LA CODEPENDENCIA EN UN GRUPO DE FAMILIARES DE PACIENTES DROGADEPENDIENTES
}

\author{
Gilmar Guevara Ortega
}

\begin{abstract}
RESUMEN
En la presente investigación se estudiarón los niveles de Autoeficacia y Codependencia de los familiares de pacientes drogadependientes que se encuentran recibiendo tratamiento en Comunidades Terapéuticas de Lima Metropolitana. La muestra está conformada por 115 familiares de pacientes que tienen problemas de dependencia a sustancias psicoactivas como alcohol, pasta básica de cocaína, clorhidrato de cocaína y marihuana. Los instrumentos utilizados fueron: La "Escala de Codependencia de Spann-Fischer" y la "Escala de Autoeficacia generalizada" de Babler-Schwarzer. Ambos nos permiten evaluar los niveles de Codependencia el primero y Autoeficacia el segundo. El análisis psicométrico se realizó en base al análisis factorial, y la consistencia interna se estableció utilizando el coeficiente de con fiabilidad Alfa de Cronbach.

Los resultados nos demuestran que existe una relación significativa entre la codependencia y la autoeficacia, no en términos de puntajes sino en términos de niveles, así como algunas variables como edad, tiempo de tratamiento y nivel de instrucción se relacionan significativamente con la codependencia. Otras como el nivel de instrucción, tiempo de internamiento tienen relación significativa con la codependencia.
\end{abstract}

Palabras Clave: Autoeficacia, Codependencia, Drogadependiente, Comunidad Terapeutica.

\begin{abstract}
In the present investigation the levels of selfefficacy and the relatives of patient drug addict Codependencia are studied that are receiving treatment in Therapeutic Communities of Metropolitan Lima. The sample this conformed by 115 relatives of patients that have dependence problems to substances like alcohol, he/she pastures basic of cocaine, hydrochlorate of cocaine and marihuana.

The instruments used for the present investigation are: The Scale of Codependency of Spann-Fischer and the Scale of widespread self-efficacy of Babler-Schwarzer. Both they allow us to evaluate the levels of Codependencia the first one and self-efficacy the second. The psicometric analysis one carries out based on the factorial analysis and the consistency goes into it established using the coefficient of dependability Alpha of Cronbach.

The results demonstrate us that a significant relationship exists between the codependency and the self-efficacy, as well as some variables as age, time of treatment and instruction level are related significantly with the codependency.

Others as the instruction level, time of internment has significant relationship with the codependency.

Words Key: self-efficacy, Codependency, Drogodependiente, Therapeutic Communitties.
\end{abstract}

Colaboradores: Miguel Escurra Mayaute, Héctor Hernández Valz, Luis Del Castillo Gamboa, Tomy Flores Lezama. 


\section{INTRODUCCION}

\section{ANTECEDENTES}

En el campo del tratamiento de la drogadependencia, el trabajo con las familias se traduce en el aprendizaje de una serie de pautas y compromisos que conducen a los integrantes del núcleo familiar a asumir comportamientos que complementen y apoyen los logros obtenidos por el drogadependiente en su tratamiento. La familia del drogadependiente debe pasar por un proceso al igual que el paciente, y es en esta situación en que la familia debe iniciar un proceso de cambio y modificación de patrones de conducta desadaptativos para poder resolver las situaciones conflictivas que se presentan en su interacción con el drogadependiente (Graña, 1986).

Cada familia asume roles y dinámicas particulares para mantener su equilibrio y armonía entre sus miembros. Cuando aparece alguna situación estresante estos roles y dinámicas tienden a distorsionarse y es en esta trastocación de roles en que cada uno de los integrantes adoptará un rol que le permita adaptarse a los eventos estresores. Uno de ellos será quien asuma actitudes de codependencia. La codependencia es definida como un comportamiento que presenta cualquier familiar de un adicto que refuerzan la negación del adicto facilitando con ello el consumo de drogas, el boicot al tratarpiento o favoreciendo la recaída del mismo. La codependencia se caracteriza por problemas en las relaciones cercanas o íntimas, factores de control regidos por locus externo y por la necesidad de tener un control sobre e1 otro, dificultades a nivel de sentimientos, negación de sí mismo, hiperresponsabilidad y utilización de la negación como mecanismo de respuesta. Es una persona que se dedica a cuidar de la otra, pues un adicto necesita de muchos cuidados, sin permitir o posibilitar que elimine su adicción.

La autoeficacia es un constructo propuesto por A. Bandura (1977), quien observó que las personas crean y desarrollan sus autopercepciones acerca de la propia capacidad de conseguir sus metas y controlar su ambiente. Bandura define la autoeficacia como: "las creencias en la propia capacidad para organizar y ejecutar las acciones requeridas para manejar las situaciones futuras, es decir la autoeficacia es la capacidad que tiene una persona de saber que puede hacer lo que está tratando de hacer.

Consideramos que las personas codependientes presentan niveles de autoeficacia disminuidos por lo que tratan de mantener las condiciones de dependencia, tanto de ellas mismas como de su familiar adicto, con la finalidad de mantener el entorno familiar disfuncional ya que no pueden desenvolverse adecuadamente en un entorno familiar sano.

\section{JUSTIF1CACIÓN}

1. La codependencia es uno de los problemas más importantes a tratar en el abordaje de la drogadependencia, por ello es importante su evaluación.

2. Al presentar niveles de autoeficacia disminuidos los codependientes impiden el avance terapéutico del drogadependiente por lo que es importante conocer su autoeficacia para facilitar el tratamiento.

3. El conocer la relación entre ambas variables -codependencia y autoeficacia- permitirá diseñar progamas de prevención y tratamiento para estas personas.

4. Evaluando la relación entre codependencia y autoeficacia nuestra posibilidad de ayudar a los pacientes adictos se verá aumentando y estaremos aliviando uno de los principales 
factores causantes de recaídas: el entorno familiar.

\section{PLANTEAMIENTO DEL PROBLEMA}

Dentro de todas las técnicas de tratamiento y rehabilitación para drogadependientes, cualquiera sea su modalidad, la terapia familiar se hace indispensable para conseguir la rehabilitación completa del individuo. La familia, primer núcleo en la reinserción social del drogadependiente, tiene una participación importante y no sólo debe acompañar al paciente sino que tiene que participar activamente en la Terapia.

Diversos estudiosos de las adicciones consiguieron tipificar los diversos roles familiares que adoptan los miembros de una familia cuando uno de sus integrantes cae en un problema adictivo. Uno de los roles más importantes fue denominado como el coadicto o codependiente. Este ha sido caracterizado como una persona que también necesita ayuda, incluso nos atreveríamos a afirmar que primero tienen que tratarse o modificar sus patrones de conducta el codependiente, para que el adicto también consiga éxito en su rehabilitación. Consideramos que las creencias de la persona codependiente son relevantes para determinar su comportamiento, una de las creencias más importantes es la Autoeficacia. Muchos de los comportamientos del codependiente son influenciados por su nivel de autoeficacia, por lo que debe existir diversas fluctuaciones en el nivel de la misma.

Deseamos determinar si los niveles de Autoeficacia de los diversos miembros de una familia varían o fluctúan entre niveles altos y bajos, y si esta variación se acompaña de comportamientos característicos de codependencia.

\section{MARCO TEÓRICO}

\section{Codependencia}

Es durante la década de los setenta que se crea el término de codependencia, primero como coadicción, para describir a la persona que mantenía una relación íntima y estrecha con una persona alcohólica, adicta o con otros trastornos psicológicos (depresión, trastornos alimenticios, problemas de conducta, etc.), lo que se observó en aquellas épocas fue que las personas que convivían junto a aquellas que tenían problemas terminaban imitando comportamientos de los mismos, así como intentaban controlar a la persona con problemas y hacían que su mundo girase alrededor de ellas. Eran las que conducían al tratamiento a sus familiares con problemas, incluso a costa de sí mismos. Vivían el tratamiento y rehabilitación de su familiar, asumiéndolo como un resarcir su propia culpabilidad pues se achacaban a ellos mismos el problema adictivo, y curiosamente cuando el adicto comenzaba a mejorar cometían desatinos con ellos que los llevaban de nuevo al consumo, reiniciándose nuevamente el ciclo mencionado.

Los inicios del término se pierden en el devenir de la historia y para hacer la historia de la codependencia hay que hacer la historia de la terapia familiar. Los alcances más cercanos son el inicio de la Terapia familiar con el movimiento creado por Virginia Satir al inicio de los años cincuenta del siglo XX; coincidentemente, por estas épocas es fundada Al-Anon, grupo de terapia familiar de Alcohólicos anónimos. Estamos en la época dorada de la corriente humanista en Psicología con Maslow, Ackerman, Bateson, Haley, Watzlawick, Rogers, Perls, entre otros que surgían y varios de ellos van a sentar las bases de la terapia 
familiar sistémica (Withfield, 1991).

Vernon Jonson, 1973, crea el término coalcoholismo, y plantea la necesidad de la intervención familiar en el tratamiento y recuperación del alcohólico. En los años 80 el término codependiente comienza a ser usado para describir cualquier acercamiento a la persona dependiente a sustancias, y de allí no tardará mucho en aparecer el término codependencia. En 1986 es fundada la Institución CoDA (Co-dependents Anonyrnous) y en 1990 es fundado el Concejo Nacional sobre Codependencia o NCC (National Council on Co-dependence), ambas instituciones en USA, que tienen como misión brindar información y recursos varios sobre codependencia y rehabilitación (C. Whitfield, 1991).

Siempre que uno de los miembros de una familia desarrolle alguna conducta adictiva existen posibilidades de que otro de los demás miembros de tal familia desarrollen un comportamiento conocido como codependencia o coadicción. La característica de la persona codependiente es presentar comportamientos contrarios a los que se propone el tratamiento; en otras palabras, mantienen la adicción de dos maneras notorias: Primero, permitiendo el avance de la dependencia desde su inicio hasta la búsqueda de ayuda, con comportamientos "psicopatoides" de negación y no aceptación, de encubrimientos y mentiras, de complicidad y engaños, de salvación y "abnegación". El codependiente primero niega el problema adictivo, una vez evidenciado lo encubre y protege, luego lo permite y alienta con conductas de complicidad y por último, considera que sólo él o ella puede salvarlo o brindarle la ayuda requerida.

En segundo término, el codependiente va a realizar un boicot, explícito o implícito, a los esfuerzos del drogadependiente por salir de su adicción. Esto se da a través de comportamientos como descalificar a los terapeutas o al centro de tratamiento, transmitir negatividad en sus mensajes al adicto, crear necesidades (reales o no) para que el adicto abandone el tratamiento, actuar como sí el adicto vaya a recaer, conducta perseguidora, entre otras. Todas estas formas de actuar son un mensaje escondido hacia el adicto: "te necesito, pero te necesito mal; si estás bien no te necesito" (J. Bradshaw, 1990).

Lo más importante de estos comportamientos es que la persona codependiente piensa, y asegura, que su intención es ayudar y solucionar el problema de la adicción de su familiar, quiere decir esto que la conducta codependiente es una forma de actividad de carácter automático que ha sido reforzada y mantenida como tal a través de los años y que connota una finalidad adaptativa dentro de la familia para tal fin. El codependiente presenta rasgos de personalidad, claramente identificables, con formas de pensar irracionales que son muy difíciles de cambiar ya que van acompañada de patrones afectivos perturbadores como angustia, ansiedad, depresión, ira incontrolable, así como baja autoestima, temor al abandono, défícit en la toma de decisiones, incapacidad de autocrítica, descontrol de impulsos, sentimiento de culpa, entre otras.

Muchos teóricos de las adicciones tipifican a las mujeres como principales codependientes y con característicos comportamientos de víctima y mártires de una relación, lo cual es cierto pero tenemos también la otra realidad de una persona que es perseguidora y salvadora del adicto, que piensa que el adicto no puede hacer nada sin él o ella y cuando se empieza a recuperar boicotea el tratamiento para recuperar su status anterior y proseguir con el dominio y la dependencia relacional (Alvarado, S, 1997). 
Lo anterior nos conduce a diferenciar la codependencia y la dependencia relacional. Se define a la codependencia como un "trastorno individual específico, configurador de la identidad del individuo, caracterizado por un intenso temor al abandono (exclusión o descalificación) y un girar en torno a otro. Su génesis se asocia a una dinámica interaccional, que surge frente a una respuesta familiar disfuncional ante un determinado tipo de enfermedad o trastorno de otro de sus miembros" (A. Coddou, M Chadwick, 1996). Dentro del grupo de las llamadas dependencias relacionales que presentan entidad propia como las denominadas dependencias emocionales: adicción al amor, interdependencia, dependencia afectiva, etc., y otras ligadas a otros trastornos de dependencia (sobre todo dependencia a sustancias psicoactivas), como la codependencia y la bidependencia. Entonces, la dependencia relacional se refiere a la adicción a personas con las que se mantiene algún vínculo -que puede ser de pareja, amistad, trabajo, consanguineidad, etc.-, ambos cuadros se pueden dar en una misma persona, permitiendo que una misma persona se "haga cargo" de otra que tiene problemas de adicción y a la vez no pueda vivir sin ella sin que esta separación le haga daño.

A partir de los años 90 se viene usando el término bidependencia o dependencia doble que se da en drogadependientes que además de su adicción a las sustancias psicotrópicas presentan dependencia relacional. Esta dependencia relacional es de tipo acomodaticio, pasivizante y suprirnidor de la autonomía, inclusive se mantiene aunque la relación pueda ser perjudicial y dañina. El adicto mantiene la relación porque "le conviene", estar con alguien a quien pueda manipular, que lo acepta sin restricciones $\mathrm{y}$, de una u otra manera le permite continuar con su vida, tal y como esta sea, (Carlos.Sirvent, 2,000) .

Los comportamientos de las personas codependientes presentan un patrón con uniformidad de respuestas y una variación de las mismas. La variación depende del vínculo que los una con el drogadependiente, es decir, serán diferentes los comportamientos de una esposa/o, padres, hermanos, suegros, hijos, etc., pero esto no implica que sean cuadros o entidades diferentes sino que son expresiones desde otros planos relacionales y que no pueden ser interpretados fuera del contexto de la relación que tiene el codependiente con el adicto. Si observamos circularmente a la familia en su dinámica relacional podríamos señalar la analogía física de las fuerzas centrífugas y centrípetas. Mientras que los demás miembros de la familia presentan una dinámica centrífuga, se alejan, física o psicológicamente del adicto; por el contrario, el codependiente mantiene sus energías centrípetas y siempre se mantiene al lado, física o psicológicamente, del adicto.

La génesis de la codependencia es de carácter multicausal (R. Vacca, 1998), pero necesariamente se tienen que dar un trastorno de tipo adictivo en algunos de los miembros de la familia. Es un requisito indispensable, o condición necesaria que el trastorno ante el cual surge la codependencia sea crónico. En esta cronicidad radica uno de las intersecciones más sólidas de la conducta codependiente, la esperanza de curación asociada a la cronicidad. La codependencia puede llegar a convertirse en una adicción más, hasta el punto de que la mayoría de las características de un codependiente van a coincidir en gran medida con las del adicto.

Las características diagnósticas de la codependencia no están claras y cada autor considera las que ha encontrado en sus investigaciones y casos clínicos. Esto es importante señalarlo ya que las escalas y cuestionarios de evaluación van a diferir en función de las características señaladas para el trastorno. Los manuales y tratados de clasificación 
diagnóstica como son el CIE-10 y el DSM III, III-R Y IV, no consideran dentro de sus categorías diagnósticas a la codependencia. Pero se han realizado varias propuestas para que se incluya la codependencia en éstos. Una de las serias propuestas más serias sobre el tema fue realizada por Cermak, (en 1986), que también es mencionado por Withfield (1991) de la forma siguiente:

\section{Criterios diagnósticos sugeridos para la codependencia}

\section{(Desorden de la Personalidad Co-dependiente)}

1. Inversión continuada de Autoestima en su capacidad para controlarse a sí mismo y a los otros para enfrentar serias consecuencias adversas.

2. Asume la responsabilidad de encargarse de las necesidades de los demás, incluso a costa de reconocer sus propias necesidades.

3. Ansiedad y dificultad para reconocer los límites acerca de la separación o intímidad con los demás.

4. Permanentemente cae en relaciones con gente que tiene desórdenes de la personalidad, dependencia a sustancias o desórdenes impulsivos individuales.

5. Presenta, por lo menos tres, de las siguientes características:

- Excesiva confianza en cosas negativas - Constricción emocional (con o sin explosiones dramáticas)

- Depresión

- Hipervigilancia

- Compulsiones

- Ansiedad

- Abuso de alcohol o drogas

- Es (o fue) víctima recurrente de abuso físico o sexual

- Presencia de estrés relacionado a una enfermedad médica

- Ha tenido relaciones con personas violentas o dependientes a sustancias (como el substituto del alcohol o droga de estas personas), por un lapso mínimo de dos años sin búsqueda de ayuda externa.

\section{Otras características que ofrecen los diferentes autores son:}

- Conducta regida por locus de control externo, creen que sus acciones y pensamientos derivan de lo que hace su familiar adicto.

- Hiper-Responsabilidad, considera que nadie hará las cosas como ellos mismos y asumirán compromisos que van más allá de sus límites.

- Sentimientos intensos de culpabilidad, muchos codependientes se consideran causantes del comportamiento adictivo de su familiar, y lo expresan y lo viven de esa manera.

- Negación de sí mismo, los problemas del adicto están antes que ellos, postergan muchos de sus proyectos en función del tratamiento que hacen los adictos.

- Búsqueda de aceptación de los demás, esta creencia irracional de que todos lo deben de apreciar marca todas sus acciones.

- Sobreprotección, se consideran a sí mismas como autosuficientes y únicas para ayudar al adicto y a éste lo consideran débil y vulnerable por ello tratan que nada ni nadie, excepto ellas mismas, se encarguen de todo.

- Manipulación, a veces es la única forma de conseguir que se haga algo por el adicto, mienten, ocultan información, o la tergiversan, para así conseguir sus propósitos. 
- Victimismo-Mártir, se consideran las únicas perjudicadas de los problemas que le ha acarreado la relación con el adicto, se consideran que son las que sufren más y a las que más daño se les ha hecho.

- Gran tolerancia al abuso recibido, muchas de las personas codependientes han sido humilladas, maltratadas, golpeadas, engañadas, ésta es una norma en la vida de ellas.

- Preocupación y dependencia excesiva, hacia otra persona, cuando no ejercen un control directo sobre la persona adicta sienten que algo les falta y están constantemente pendientes de ellos.

- Temor excesivo al abandono y al rechazo, y por evitar estos sentimientos están dispuestas a hacer cosas más allá de sus límites.

- Inseguridad y falta de confianza en sí mismos, constantemente están abordando a otras personas con preguntas acerca de su problemática y es esta inseguridad lo que no les permite avanzar en el tratamiento.

- Rigidez, dificultad para aceptar cambios, cualquier modificación de su rutina psicológica crea en estas personas un conflicto grande generándole sentimientos de intranquilidad y depresión.

- Deshonestidad, engaños, mentiras, como es parte de la conducta manipulatoria.

- Supresión e inversión de valores.

- Déficit de asertividad, son incapaces de expresar adecuadamente sus opiniones y por ello acumulan molestias e incómodos.

- Comportamiento Salvador-rescatador, se consideran que son las únicas (y muchas veces las mejores) personas que pueden sacar al adicto del problema en que se halla.

Es probable que cada persona que trabaja en drogadependencia retire o incluya algunas características, esto es válido ya que este concepto aún no ha quedado claramente establecido. En estos tiempos la codependencia es abordada por dos tipos de literatura, la popular y la académica. Debido a que no ha sido reconocido categóricamente, como una disfunción o patología, dentro de la gnoseología psiquiátrica, es que la codependencia es uno de los términos más abordados por la literatura popular de autoayuda. Por ello encontramos también que cada autor propone un concepto diferente y muy particular de lo que es la codependencia y por la misma razón cada autor nos señalará las características que él considere relevantes de acuerdo a su experiencia y a su enfoque teórico. Por ello es necesario que se asuma un acuerdo y una tipificación en el concepto y las características diagnósticas (Haaken, 1990; Cermak, 1986).

\section{AUTOEFICACIA}

La autoeficacia es un término creado por Albert Bandura para referirse al conjunto de creencias y autopercepciones que tenemos todas las personas acerca de nuestra propia capacidad para conseguir metas y controlar nuestro ambiente, es nuestra creencia de que podemos hacer lo que estamos tratando de hacer.

Este conjunto de creencias es importante para nuestro real funcionamiento y realización de la actividad humana, afecta a todos los niveles ejecutivos de la persona, desde los afectivos, motivacionales, hasta los cognitivos y racionales. En suma, la autoeficacia va a determinar nuestra perfomance adaptativa ante las diversas situaciones estresantes que enfrentamos día a día. 
La autoeficacia vendría a ser uno de los tipos de pensamientos o creencias autorreferenciales que controlan nuestro comportamiento. Bandura (1986) sostiene que es la propia autoeficacia la que afecta nuestra elección de actividades, afecta al esfuerzo que ponemos en ellas y mantiene o no nuestra

constancia en la ejecución de tales actividades. Sostiene que existen cuatro fuentes principales que producen la autoeficacia:

1. Las experiencias anteriores

2. Las experiencias vicarias

3. La persuasión verbal, y

4. Los estados fisiológicos

Las experiencias anteriores son la principal fuente de la Autoeficacia y es la que tiene una gran influencia en nuestro comportamiento. Todos juzgamos nuestros actos en función de los éxitos o fracasos obtenidos. Si algo que hacemos tiene éxito o buen resultado, aumentará nuestra autoeficacia; por el contrario, nuestros fracasos la disminuirán.

Las experiencias vicarias se refieren a la observación de la conducta de los demás, al modelado que ejercen las demás personas hacia nosotros. Siendo parte del Aprendizaje Observacional o social, Bandura ha establecido claramente los principios que sostienen este tipo de aprendizaje.

La persuasión verbal se refiere a la persuasión social, al efecto que ejerce el lenguaje sobre nosotros, nuestra autoeficacia nace y se desarrolla de acuerdo a lo que nos van diciendo las demás personas de nuestro entorno social, sobre todo lo que nos dicen las personas significativas.

También puede variar nuestra eficacia en función de la percepción de nuestros estados orgánicos. Las reacciones vegetativas de la ansiedad o estrés serán siempre asociadas a percepciones de autoeficacia pobres, el hecho de saber que uno se encuentra ruborizado al exponer un tema será asociado a situaciones en donde el desempeño sea pobre.

Así como hay fuentes de Autoeficacia, existen factores que afectan a la misma. Estos factores son:

1. El establecimiento de metas

2. El procesamiento de la información

3. Los modelos

4. La retroalimentación, y

5. Los premios

Las creencias de autoeficacia no son únicas, invariables y permanentes, sino que se presentan a lo largo de tres dimensiones:

1. Magnitud

2. Fuerza

3. Generalidad

La magnitud de la autoeficacia implica la cantidad de cosas que una persona se considera capaz de hacer, la cantidad de retos que puede superar, el número de pasos que tiene que realizar para alcanzar una meta. 
La fuerza está referida al convencimiento y resolución de una persona respecto a lo que puede o no hacer.

La generalidad comprende el factor transituacional que tienen nuestras conductas de éxito o fracaso. Es decir, si éstas se extienden o no a otras situaciones o contextos.

La Autoeficacia está muy ligada al concepto de control, y como hemos podido ver las personas codependientes siempre ejercen, bien o mal, alguna forma de control hacia las personas que las rodean. Se puede ejercer un control directo sobre las personas o un control indirecto, este último se ejerce a través de otras personas. Otra forma de clasificar el control es la siguiente: Control primario, se refiere al control que se ejerce sobre el ambiente exterior de la persona, es cuando nuestro esfuerzo se aboca a modificar nuestro entorno. Control secundario, aquí el esfuerzo desplegado es hacia nosotros mismos, no modificamos nuestra realidad existente, sino cambiamos nosotros, en cualquier aspecto de nuestro mundo psicológico para adaptamos a las demandas del ambiente, (Canto y Rodríguez, J., Autoeficacia y educación)

Baessler y Schwarzer (1996) consideran a la autoeficacia como el factor básico para el afrontamiento, siempre evaluamos nuestras acciones, pensamient9s y sentimientos, buscando nuestras 'fortalezas y debilidades en los mismos. El nivel de autoeficacia alcanzado determinará nuestra estrategia y estilo de afrontamiento. Las personas que tienen un elevado nivel de autoeficacia ofrecerán un mayor esfuerzo en sus actividades y tienen mayor persistencia en la ejecución de las mismas. Mantienen sus metas y continúan hacia ellas a pesar de algunos fracasos que puedan encontrar en el camino. Se podría decir, que las personas de alta eficacia son resilientes, enfrentan sus retos, buscan solucionar sus problemas y acepta los desafíos que se les presentan.

\section{ESTUDIOS PREVIOS}

Malpartida, 1998, trabajó con una muestra clínica de 90 coadictos de diversas comunidades terapéuticas de Lima, utilizando un Cuestionario, que fue validado en este estudio, encontró que las conductas codependientes están más relacionadas al género femenino. Encontró además conductas problemas en el $74 \%$ de su muestra.

Delgado D., Y Pérez A., (Colombia, 2001), trabajaron con 301 sujetos, de los cuales 151 tenían alguna relación afectiva con un consumidor de drogas y los 150 restantes no utilizaron un instrumento diseñado por los autores basado en el CODAT de HughesHarnmer (1998), además aplicaron dos escalas, de Autoestima y de Relaciones familiares, así, como el Cuestionario de Depresión de Beck. Encontraron diferencias entre hombres y mujeres, presentando ellas mayor depresión, baja autoestima y control emocional. En la comparación intergrupos encontraron que en el grupo de codependientes eran en control emocional, focalización en el otro y necesidad de ayuda.

\section{METODOLOGÍA}

\section{HIPOTESIS}

H1 "Existe una relación significativa entre los niveles de autoeficacia y la codependencia en las familias de pacientes drogadependientes".

\section{OBJETIVOS}


1. Evaluar los niveles de codependencia aplicando la "Escala de Codependencia de SpannFischer".

2. Evaluar los niveles de autoeficacia con la "Escala de Autoeficacia generalizada" de Babler- Schwarzer.

3. Establecer las relaciones existentes entre la codependencia y la autoeficacia a través de los resultados de ambas escalas.

\section{DISEÑO DE INVESTIGACIÓN}

El diseño de la investigación utilizado fue el descriptivo comparativo, pues se compararon los resultados de dos mediciones, autoeficacia y codependencia.

\section{POBLACIÓN Y MUESTRA}

La población estuvo conformada por familiares de pacientes drogadependientes que se encuentran asistiendo a terapia familiar de apoyo en las Comunidades Terapéuticas de Lima Metropolitana.

La muestra está conformada por 115 personas obtenidas siguiendo las pautas del diseño muestral no probabilístico de tipo intencional. Este tipo de selección se basa en el conocimiento que el investigador posee de la población y sobre ellos se identifica a los grupos considerados representativos para ser incluidos en la muestra (Alarcón, 1991). Los centro utilizados para la conformación de la muestra fueron las Comunidades terapéuticas "Escuela de Vida" y "Ayudémonos" y el Hospital Víctor Larco Herrera (Pabellón 18).

\section{INSTRUMENTOS DE RECOLECCIÓN DE DATOS}

En la presente investigación se aplicarán:

La "Escala de Codependencia de SpannFischer" y la "Escala de Autoeficacia generalizada" de Babler-Schwarzer. Con las cuales se realizará lo siguiente:

a) Revisión del lenguaje y redacción de las Escalas.

b) Aplicación Piloto

c) Evaluación de la validez de constructo de las escalas, en base al análisis factorial

d) Cálculo de la Confiabilidad, en base al Coeficiente Alfa de Cronbach.

IV. RESULTADOS

1. COMPOSICIÓN DE LA MUESTRA DE ACUERDO A LAS VARIABLES ESTUDIADAS

TABLA N $\mathbf{N}^{\circ} 1$

DISTRIBUCIÓN DE LA MUESTRA SEGÚN SU CENTRO DE PROCEDENCIA

\begin{tabular}{ccc} 
CENTRO DE PROCEDENCIA & FRECUENCIA & PORCENTAJE \% \\
\hline CT Escuela de Vida & 62 & 54 \\
CT Ayudémonos & 17 & 15 \\
H. Larco Herrera & 36 & 31 \\
\hline Total & 115 & 100
\end{tabular}

TABLA $\mathbf{N}^{\circ} 2$ 


\section{DISTRIBUCIÓN DE LA MUESTRA SEGÚN EDAD}

\begin{tabular}{ccc} 
VARIABLE EDAD & FRENCUENCIA & PORCENTAJES \% \\
\hline $18-22$ & 10 & 8.7 \\
$23-30$ & 28 & 24.3 \\
$31-40$ & 15 & 13 \\
$41-72$ & 62 & 53.9 \\
\hline Total & 115 & 100
\end{tabular}

TABLA N $\mathbf{N}^{\circ}$

DISTRIBUCIÓN DE LA MUESTRA SEGÚN SU GRADO DE INSTRUCCIÓN

\begin{tabular}{ccc} 
VARIABLE INSTRUCCIÓN & FRECUENCIA & PORCENTAJE \% \\
Primaria & 21 & 18.3 \\
Secundaria & 53 & 46 \\
Superior & 41 & 35.7 \\
\hline Total & 115 & 100
\end{tabular}

TABLA $\mathrm{N}^{\circ} 4$

COMPOSICIÓN DE LA MUESTRA POR CONSUMO DE SUSTANCIA DE SU FAMILIAR

\begin{tabular}{ccc} 
VARIABLE SUSTANCIA & FRENCUENCIA & PORCENTAJE\% \\
Alcohol & 12 & 10.4 \\
Cocaina & 39 & 33.9 \\
Pasta Básica & 56 & 48.7 \\
Marihuana & 8 & 7 \\
\hline Total & 115 & 100
\end{tabular}

TABLA $\mathbf{N}^{\circ} 5$

COMPOSICIÓN DE LA MUESTRA POR TIEMPO DE CONSUMO

QUE TIENE EL FAMILIAR DROGADEPENDIENTE

\begin{tabular}{ccc} 
VARIABLE T. CONSUMO & FRECUENCIA & PORCENTAJE\% \\
$($ Años) & & \\
$0-1$ & 2 & 1.7 \\
$1-3$. & 8 & 7 \\
$3-6$. & 23 & 17.8 \\
$6-10$. & 49 & 20 \\
$10-20$. & 16 & 42.6 \\
20 a más & & 13.9 \\
\hline $\mathbf{N}=\mathbf{1 1 5}$ & &
\end{tabular}

TABLA $\mathbf{N}^{\circ} 6$ 


\section{COMPOSICIÓN DE LA MUESTRA POR EL TIEMPO \\ DE TRATAMIENTO DEL DROGADEPENDIENTE}

\section{TIEMPO DE TRATAMIENTO}

0 - 6 meses

$6-12$ meses

1 - 2 años

más de dos años

\section{FRECUENCIA}

30

34

29

22

\section{PORCENTAJE\%}

26.1

29.6

25.2

19.1

\section{$\mathbf{N}=\mathbf{1 1 5}$}

TABLA N $\mathbf{N}^{\circ}$

COMPOSICIÓN DE LA MUESTRA POR EL NÚMERO DE TRATAMIENTOS DEL DROGADEPENDIENTE

\begin{tabular}{ccc} 
NÚMERO DE TRATAMIENTOS & FRECUENCIA & PORCENTAJE\% \\
1 & 75 & 65.2 \\
2 & 26 & 22.6 \\
3 & 8 & 7 \\
4 & 4 & 3.5 \\
Más de 4 & 2 & 1.7 \\
\hline
\end{tabular}

$\mathbf{N}=\mathbf{1 1 5}$

\section{ANÁLISIS ESTADÍSTICO DE LOS INSTRUMENTOS}

\section{Tabla $\mathrm{N}^{\circ} 1$}

La mayoría de los familiares corresponden a comunidades terapéuticas (Escuela de Vida y Ayudémonos) con el 85\%; el resto de la muestra procede de familias de internos de un centro hospitalario $15 \%$.

\section{Tabla N ${ }^{\circ} 2$}

E153.9 \% de la muestra de familias tienen 41 o más años de edad; al contrario los jóvenes sólo constituyen el $8.70 \%$ entre los 18 y 22 años.

\section{Tabla $\mathrm{N}^{\circ} 3$}

La mayoría de las familias tienen instrucción secundaría y superior, acumulando ambas el $81 \%$, mientras que sólo el $18 \%$ tienen educación primaria.

\section{Tabla $N^{\circ} 4$}

El mayor porcentaje de consumidores de pasta básica de cocaína representa el $48.7 \%$ y los consumidores de clorohidrato de cocaína el $33.9 \%$.

Tabla $\mathrm{N}^{\circ} 5$

El $54.5 \%$ de consumidores tienen más de 10 años de consumo y el 8.7 
$\%$ tienen menos de 3 años de consumo.

Tabla $\mathrm{N}^{\circ} 6$

Al ser familiares de reincidentes en tratamiento se observa por los intervalos de tiempo que son homogéneos en función al tiempo de tratamiento.

\section{Tabla $\mathrm{N}^{\circ} 7$}

El $65.2 \%$ de los familiares tienen a sus pacientes en un primer tratamiento, el $22.6 \%$ en segundo tratamiento y sólo el $5.2 \%$ tienen a sus familiares en cuatro o más tratamientos.

TABLA $\mathbf{N}^{\circ} 8$

\section{ANÁLISIS PSICOMÉTRICO DE LA ESCALA DE AUTOEFICACIA}

\begin{tabular}{cccc} 
ITEM & M & DS & ritc \\
1 & 3.51 & 0.88 & $0,49^{*}$ \\
2 & 3.62 & 0.81 & $0,62^{*}$ \\
3 & 3.3 & 0.94 & $0,62^{*}$ \\
4 & 3.24 & 0.89 & $0,64^{*}$ \\
5 & 3.45 & 0.76 & $0,60^{*}$ \\
6 & 3.09 & 0.96 & $0,59^{*}$ \\
7 & 3.32 & 0.92 & $0,65^{*}$ \\
8 & 3.67 & 0.6 & $0,48^{*}$ \\
9 & 3.36 & 0.81 & $0,43^{*}$ \\
10 & 3.49 & 0.81 & $0,61^{*}$ \\
\hline
\end{tabular}

ALFA DE CONBACH $=0,86$

*significativo $r>0,20$

$\mathbf{N}=\mathbf{1 1 5}$ 


\section{TABLA $\mathbf{N}^{\circ} 9$ \\ ANÁLISIS PSICOMÉTRICO DE LA ESCALA DE CODEPENDENCIA}

\begin{tabular}{cccc} 
ITEM & M & DS & ritc \\
1 & 3.44 & 1.85 & $0,39^{*}$ \\
2 & 3.54 & 1.77 & $0,32^{*}$ \\
3 & 3.62 & 1.95 & $0,29^{*}$ \\
4 & 3.07 & 1.85 & $0,33^{*}$ \\
5 & 3.17 & 1.92 & $0,33^{*}$ \\
6 & 2.55 & 1.98 & $0,51^{*}$ \\
7 & 3.36 & 1.85 & $0,25^{*}$ \\
8 & 4.17 & 1.94 & $0,36^{*}$ \\
9 & 4.14 & 1.83 & $0,42^{*}$ \\
10 & 4.19 & 1.64 & $0,44^{*}$ \\
11 & 3.14 & 1.91 & $0,35^{*}$ \\
12 & 3.32 & 1.97 & $0,20^{*}$ \\
13 & 4.55 & 1.78 & $0,34^{*}$ \\
14 & 4.33 & 1.75 & $0,49^{*}$ \\
15 & 3.83 & 1.83 & $0,47^{*}$ \\
16 & 4.36 & 1.71 & $0,45^{*}$ \\
\hline ALA DECONBACH
\end{tabular}

ALFA DE CONBACH $=0,77$

*significativo $r<0,20$

$\mathbf{N}=\mathbf{1 1 5}$

De acuerdo a los resultados obtenidos por la prueba de autoeficacia (ver tabla no.8), se aprecia que todos los ítems son válidos, pues alcanzan correlaciones ítem-test superiores al criterio de 0.20. Además se observa que se alcanza una confiabilidad a través del coeficiente Alfa de Cronbach de 0.86, lo que corrobora que la prueba de Autoeficacia es confiable.

En relación a la prueba de Correlaciones ítem-test superiores al criterio de 0.20 . También se encuentra la confiabilidad a través del coeficiente alfa de Cronbach es de 0.77, lo que permite concluir que la prueba de Codependencia es confiable.

TABLA N $\mathbf{N}^{\circ} 10$

PRUEBA DE BONDAD DE AJUSTE A LA CURVA NORMAL DE LAS VARIABLES ESTUDIADAS

\begin{tabular}{cccc} 
VARIABLE & M & DS & K-SZ \\
\hline AUTOEFICACIA & 30.74 & 3.67 & $1.70 * *$ \\
\hline $\begin{array}{c}\text { CODEPENDENCIA } \\
\text { ** }<\text {,01 }\end{array}$ & 58.78 & 14.02 & 0.96 \\
\hline $\mathbf{N}=\mathbf{1 1 5}$ & & & \\
\hline
\end{tabular}

El análisis de la bondad de ajuste a la curva normal, realizado a través de la prueba de Kolmogorov-Smirnov indica que existen diferencias significativas en la Autoeficacia, lo que indica que en este constructo sólo se pueden aplicar estadísticas No paramétricas, en 
tanto que en la Codependencia si existe una adecuada aproximación a la curva normal, por lo que es factible utilizar en ese caso estadísticas Paramétricas.

\section{ANALISIS DE LA CORRELACION}

El análisis realizado a través de la correlación de Spearman entre la autoeficacia y la codependencia, indica que se obtiene una $\mathrm{r}=0.10$, la cual no es estadísticamente significativa y presenta una baja correlación entre ambos constructos.

TABLA $\mathbf{N}^{\circ} 11$

ANÁLISIS DE TABULACION CRUZADA DEL NIVEL DE CODEPENDENCIA y EL NIVEL DEAUTOEFICACIA

\begin{tabular}{lccccc} 
& & BAJO & MEDIO & ALTO & TOTAL \\
\hline & BAJO & 17 & 13 & 13 & 43 \\
& MEDIO & 18 & 15 & 7 & 40 \\
AUTOEFICACIA & ALTO & 7 & 8 & 17 & 32 \\
& TOTAL & 42 & 36 & 37 & 115 \\
$\mathbf{X 2}=\mathbf{1 0 . 7 8}$ & & & & & \\
$\mathbf{G L}=\mathbf{4}$ & & & & & \\
$\mathbf{P}$ a dos colas $=\mathbf{. 0 2 9}$ & & & & &
\end{tabular}

El análisis de la tabulación cruzada, indica que existe una relación significativa entre los niveles de codependencia y los niveles de autoeficacia.

\section{ANÁLISIS DE LAS VARIABLES SELECCIONADAS EN RELACIÓN A LA CODEPENDENCIA y LA AUTOEFICACIA}

TABLA N ${ }^{\circ} 12$

ANÁLISIS DE VARIANZA DE UN FACTOR DE LA CODEPENDENCIA POR GRUPO DE EDAD

\begin{tabular}{ccccc}
\hline \multicolumn{5}{c}{ EDAD } \\
\hline $\mathbf{1 8}-\mathbf{2 2}$ & $\mathbf{2 3}-\mathbf{3 0}$ & $\mathbf{3 1} \mathbf{- 4 0}$ & $\mathbf{4 1}-\mathbf{7 2}$ & $\mathbf{F}$ \\
$\mathbf{N}=\mathbf{1 0}$ & $\mathbf{N}=\mathbf{2 8}$ & $\mathbf{N}=\mathbf{1 5}$ & $\mathbf{N}=\mathbf{6 2}$ & $14,22^{* * *}$ \\
\hline 55.8 & $51,10 \mathrm{a}$ & $47,87 \mathrm{~b}$ & $63,37 \mathrm{ab}$ & \\
\hline \multirow{7}{*}{$\mathrm{p}<, 001$} &
\end{tabular}

El análisis de varianza de un factor indica que existen diferencias estadísticas significativas en la codependencia según el grupo de edad, notándose que los participantes del grupo entre 41 y 71 años superan $(\mathrm{M}=65.37)$ a los que tienen entre 23 y 30 años $(M=51.10)$ y los que tienen entre 31 y 40 años $(M=47.87)$. 
TABLA N $\mathbf{N}^{\circ} 13$ ANALISIS DE VARIANZA DE UN FACTOR DE LA CODEPENDENCIA POR
GRADO DE INSTRUCCIÓN

\begin{tabular}{cccc}
\multicolumn{3}{c}{ INSTRUCCIÓN } & \multirow{2}{*}{ F } \\
\cline { 1 - 3 } PRIMARIA & SECUNDARIA & $\begin{array}{c}\text { SUPERIOR } \\
\mathbf{N = 4 1}\end{array}$ \\
\hline $\mathbf{N = 2 1}$ & $\mathbf{N = 5 3}$ & $52,02 \mathrm{a}$ & $8,90 * * *$ \\
\hline 60.19 & $63,45 \mathrm{a}$ & &
\end{tabular}

$* * * \mathrm{p}<.001$

Comparaciones significativas a través del test de Schelfle $=\mathbf{a}$

El análisis de la codependencia por grado de instrucción indica que existen diferencias estadísticas significativas, observándose los participantes con instrucción secundaria alcanzan un valor mayor $(M=63.45)$ que los de instrucción superior $(M=52.02)$.

\section{TABLA $N^{\circ} 14$ \\ ANÁLISIS DE VARIANZA DE UN FACTOR DE LA CODEPENDENCIA POR TIEMPO DE TRATAMIENTO}

\begin{tabular}{ccccc}
\hline \multicolumn{5}{c}{ TIEMPO DE TRATAMIENTO } \\
\hline $\begin{array}{c}\mathbf{0}-\mathbf{6} \text { meses } \\
\mathbf{N}=\mathbf{3 0}\end{array}$ & $\mathbf{6}-\mathbf{1 2}$ meses & $\mathbf{1}$ a 2 años & más de $\mathbf{2}$ años & $\mathbf{F}$ \\
$\mathbf{N}=\mathbf{3 4}$ & $\mathbf{N = \mathbf { 2 9 }}$ & $\mathbf{N = \mathbf { 2 2 }}$ & \\
\hline $65,43 \mathrm{ab}$ & 58.94 & $58.28 \mathrm{a}$ & $54.09 \mathrm{~b}$ & $3,97 * * *$ \\
\hline$* * \mathbf{p}<\mathbf{0 , 1}$ & & & &
\end{tabular}

Comparaciones significativas a través del test de Schelfle =:a.b

En lo que concierne a la comparación de la codependencia por tiempo de tratamiento, se encuentra que existen diferencias significativas, encontrándose que la mayor codependencia se presenta en el grupo de familias que tiene a su paciente en tratamiento de 0 a 6 meses $(\mathrm{M}=65.43)$, que superan a los de 6 a 12 meses (58.94) y los de 1 a 2 años (55.28)

TABLA N ${ }^{\circ} 15$

ANÁLISIS DE VARIANZA DE UN FACTOR DE LA CODEPENDENCIA POR SUSTANCIA

\begin{tabular}{ccccc}
\hline & \multicolumn{5}{c}{ SUSTANCIA } \\
\hline Alcohol & Cocaína & Pasta Básica & Marihuana & $\mathbf{F}$ \\
\hline $\mathbf{N = 1 2}$ & $\mathbf{N = 3 9}$ & $\mathbf{N}=\mathbf{5 6}$ & $\mathbf{N}=\mathbf{8}$ & \\
\hline 59.67 & 56.62 & 61.14 & 58.78 & 1.60 \\
\hline
\end{tabular}

No existen diferencias estadísticas significativas en los niveles de codependencia cuando el paciente haya recaído o no. 
TABLA $\mathbf{N}^{\circ} 16$

PRUEBA T DE STUDENT SOBRE LA VARIABLE SI RECAYÓ O NO EL TRATAMIENTO

\begin{tabular}{|c|c|c|c|c|}
\hline \multicolumn{2}{|c|}{$\underset{N=75}{S I}$} & \multicolumn{3}{|c|}{ NO } \\
\hline M & DS & M & DS & \\
\hline 58.43 & 15.06 & 59.45 & 11.97 & -0.37 \\
\hline
\end{tabular}

TABLA $\mathbf{N}^{\circ} 17$

ANÁLISIS DE VARIANZA POR RANGOS DE KRUSKALL-WALLIS DE LA AUTOEFICACIA POR EDAD

\begin{tabular}{|c|c|c|c|c|}
\hline & \multicolumn{3}{|c|}{ EDAD } & \multirow{4}{*}{$\mathbf{X}^{2}$} \\
\hline 178-22 & $23-50$ & $31-40$ & $41-72$ & \\
\hline $\mathbf{N}=\mathbf{1 0}$ & $N=\mathbf{2 8}$ & $N=15$ & $N=62$ & \\
\hline MR & MR & MR & MR & \\
\hline 53.95 & 64.23 & 59.6 & 55.45 & 154 \\
\hline
\end{tabular}

No existen diferencias estadísticas significativas en la Autoficacia según el grupo de edad.

TABLA $\mathrm{N}^{\circ} 18$

ANÁLISIS DE VARIANZA POR RANGOS DE KRUSKALL-WALLIS DE LA AUTOEFICACIA POR GRADO DE INSTRUCCIÓN

\begin{tabular}{cccc}
\hline \multicolumn{4}{c}{ INSTRUCCIÓN } \\
\hline PRIMARIA & SECUNDARIA & SUPERIOR & \\
$\mathbf{N}=\mathbf{2 1}$ & $\mathbf{N}=\mathbf{5 3}$ & $\mathbf{N}=\mathbf{4 1}$ & $\mathbf{X}^{\mathbf{2}}$ \\
MR & MR & MR & \\
\hline $33,76 \mathrm{ab}$ & $57,81^{\mathrm{a}}$ & $70,66 \mathrm{~b}$ & $17,21 * * *$ \\
\hline
\end{tabular}

comparación Significativa a través de la U de Mann Whithney : a,b

Existen diferencias estadísticas significativas en la autoeficacia según el grado de instrucción, apreciándose que los participantes con instrucción superior $(\mathrm{MR}=70.66)$ y con secundaria $(\mathrm{MR}=57.81)$ presentan mejores niveles de autoeficacia que los de Primaria $(\mathrm{MR}=33.76)$. 
TABLA N ${ }^{\circ} 19$

ANÁLISIS DE VARIANZA POR RANGOS DE KRUSKALL-WALLIS DE LA AUTOEFICACIA POR TIEMPO DE TRATAMIENTO

\begin{tabular}{ccccc}
\hline \multicolumn{5}{c}{ TIEMPO DE TRATAMIENTO } \\
\hline $\mathbf{0}-\mathbf{6}$ meses & $\mathbf{6}-\mathbf{1 2}$ meses & $\mathbf{1}-\mathbf{2}$ años & más de $\mathbf{2}$ años & \multirow{2}{*}{$\mathbf{2}$} \\
$\mathbf{N}=\mathbf{3 0}$ & $\mathbf{N}=\mathbf{3 4}$ & $\mathbf{N}=\mathbf{2 9}$ & $\mathbf{N}=\mathbf{2 2}$ & $\mathbf{X}^{\mathbf{2 2}}$ \\
$\mathbf{M R}$ & $\mathbf{M R}$ & $\mathbf{M R}$ & $\mathbf{M R}$ & \\
\hline $71,10 \mathrm{a}$ & $62,96 \mathrm{~b}$ & $38,10 \mathrm{abc}$ & $59,73 \mathrm{c}$ & $15.76^{* *}$ \\
$* * \mathbf{p}<. \mathbf{0 1}$ & & & \\
comparación Significativa a través de la $\mathbf{U}$ de Mann Whithney :a,b,c
\end{tabular}

El análisis por tiempo de tratamiento indica que existen diferencias estadísticas significativas en la auto eficacia, apreciándose que los familiares participantes con su paciente de $\mathrm{O}$ a 6 meses ( $\mathrm{MR}=71.10)$, los de 6 a 12 meses $(\mathrm{MR}=62.29)$ y los de más de 2 años $(M R=59.73)$, superan a los que tienen de 1 a2 años $(M R=38.10)$ de tratamiento.

TABLA $\mathbf{N}^{\circ} \mathbf{2 0}$

ANÁLISIS DE VARIANZA POR RANGOS DE KRUSKALL-WALLIS DE LA AUTOEFICACIA POR SUSTANCIA DE CONSUMO

\begin{tabular}{|c|c|c|c|c|}
\hline & & SUSTANCIA & & \\
\hline $\begin{array}{c}\text { Alcohol } \\
\mathbf{N}=12 \\
\text { MR }\end{array}$ & $\begin{array}{c}\text { Cocaina } \\
\mathbf{N}=39 \\
\text { MR }\end{array}$ & $\begin{array}{c}\text { Pasta Básica } \\
\text { N }=56 \\
\text { MR }\end{array}$ & $\begin{array}{c}\text { Marihuana } \\
\text { N = 8 } \\
\text { MR }\end{array}$ & $\mathbf{X}^{2}$ \\
\hline $36,67 \mathrm{abc}$ & $63,28 \mathrm{a}$ & $55,59 \mathrm{~b}$ & $81,63 c$ & $10,14 * *$ \\
\hline
\end{tabular}

El análisis según el tipo de sustancia consumida indica que existen diferencias estadísticas significativas en la autoeficacia, notándose que aquellos familiares de los que consumen Cocaína ( $M R=63.28)$, Pasta Básica $(M R=55.59)$ y Marihuana( $M R=81.63)$, superan a los que consumen Alcohol (MR=36.67).

\section{DISCUSIÓN.}

El presente trabajo podrá servir de punto de partida para elaborar proyectos que podrán profundizar. La revisión de la bibliografía sobre el tema ha permitido detectar las características relevantes del comportamiento del codependiente. El análisis estadístico ha permitido validar ambos instrumentos utilizados lo que posibilita una herramienta de trabajo para los profesionales de la salud que trabajen conductas adictivas.

A nivel teórico ha permitido consolidar una noción respecto a que la autoeficacia es alta en poblaciones de codependientes debido a una gran necesidad de control y la focalización en la otra persona. No se encontraron diferencias significativas en términos de puntajes (Correlación de Speatman), pero si se encuentran diferencias significativas en función de 
los niveles alto, medio y bajo. Esta incidencia de elevada autoeficacia a medida que aumenta la codependencia implica que las personas codependientes que acuden acompañando a los drogadependientes tienen muy en claro lo que deben y pueden hacer, claro que a su manera, esto podrá redundar en que la ayuda terapéutica canalice dichas potencialidades del codependiente y no se les trate como personas que no saben solucionar problemas. Sino por el contrario tienen una buena capacidad de solucionar problemas, quizás lo que les falte es la información adecuada para llevar a cabo su ayuda efectiva.

El estudio confirma una suposición clínica (Cermak, 1986) que el factor edad es determinante ya que las personas mayores presentan conductas codependientes respecto a las personas más jóvenes. También encontramos un nivel elevado de codependencia en familiares que inician un tratamiento, esto también se relaciona con un elevado nivel de autoeficacia, quizás sea producto de la motivación inicial que tienen las familias en los primeros momentos de internamiento, pero los niveles de autoeficacia son menores luego del primer año de tratamiento, considerando la duración promedio de tratamientos residenciales en nuestro país (un año en promedio), es importante considerar que esta disminución de la autoeficacia coincide con la salida del drogadependiente luego de su internamiento. Muestran mayor nivel de codependencia los que tienen instrucción secundaria sobre los de instrucción superior.

Uno de los datos más relevantes encontrados y que confirma el trabajo de los centros de atención especializada de Lima es que los niveles de codependencia no tienen ninguna relación significativa entre las diversas sustancias de consumo utilizadas por los drogadependientes, sea alcohol, pasta básica, cocaína o marihuana. Pero si hemos encontrado diferencias en la Autoeficacia, encontrando una autoeficacia reducida en los familiares de alcohólicos, esto se deba posiblemente a los factores relacionados con la sustancia ya que el consumo de alcohol es generalizado y tiene un mecanismo de control difícil de realizar, pues los estímulos desencadenantes son numerosos y además por la situación de legalidad o permisividad a la sustancia.

Los niveles de codependencia no tienen que ver tampoco con las recaídas de los pacientes durante el tratamiento, no podríamos afirmar si la presencia de recaídas aumenta o disminuye el nivel de codependencia de las familias.

\section{CONCLUSIONES}

1. El presente estudio ha permitido la validación de dos escalas -Spann-Fischer de codependencia y la de Barber-Schwarser de Autoeficacia-, demostrándose que pueden ser utilizadas en poblaciones clínicas y con un alto grado de validez.

2. El análisis de la bondad de ajuste a la curva normal, realizado a través de la prueba de Kolmogorov-Smirnov indica que existen diferencias significativas en la Autoeficacia, lo que indica que en este constructo sólo se pueden aplicar estadísticas No paramétricas, en tanto que en la Codependencia si existe una adecuada aproximación a la curva normal, por lo que es factible utilizar en ese caso estadísticas Paramétricas.

3. Se ha podido determinar que existe una relación significativa entre los niveles de autoeficacia y codependencia, a pesar de que no existe una relación significativa en términos de puntajes, mostrándose que a niveles de Codependencia altos puede encontrarse niveles de autoeficacia altos también. Con esto decimos que a pesar de que siempre se ha percibido a una persona codependiente como un saboteador del tratamiento debemos considerar que estas personas tienen un nivel de autoconfianza que puede ser útil si es bien canalizado por los psicoterapeutas.

4. En cuanto a las variables estudiadas hemos encontrado una relación entre los niveles 
elevados de codependencia y la edad, el nivel de instrucción secundario, el tiempo del tratamiento.

5. No encontramos relaciones significativas entre la codependencia y la sustancia consumida por el familiar drogadependiente y tampoco con la presencia de recaídas.

6. En cuanto a la autoeficacia hemos encontrado relaciones significativas entre los niveles de autoeficacia y el grado de instrucción, a mayor grado de instrucción mayor nivel de autoeficacia.

7. También se encuentra un mayor nivel de autoeficacia en los familiares de consumidores de drogas ilegales (cocaína, PBC y marihuana) comparados con una droga legal como el alcohol.

8. Muestran también una Autoeficacia los familiares que inician el tratamiento y mantienen estos niveles altos durante un año de tratamiento, luego de este año la Autoeficacia decae significativamente. Esto coincide con la duración de la mayoría de los centros de internamiento de nuestro país.

9. No se han encontrado diferencias estadísticas significativas en la Autoficacia según el grupo de edad.

10. Es importante considerar, para los terapeutas de familia, las variables psicológicas estudiadas, ya que ello redundará en un trabajo integral y positivo hacia los codependientes quienes han sido vistos únicamente como personas enfermas $\mathrm{y}$, muchas veces, como causantes del comportamiento adquisitivo.

\section{REFERENCIAS}

Alarcón, R. (1991). Métodos y diseños de investigación del comportamiento. Lima. Fondo Editorial de la UPCH.

Alvarado, S, (1997).www.advance.com.ar/ usuarios/acta/la\%20codependencia.

Beattie, M. Libérate de la codependencia. Editorial Sirio S.A. Buenos Aires 1998.

Bradshaw, J. (1990) Homecoming: Reclaiming and Championing Your Inner Child (Bantam).

Canto y Rodríguez, J., Autoeficacia y educación.: anguiano@tunku.uady.mx.

Cerrnack, T. L. Co-dependence, diagnosing and treating, Johnson Institute Books, Minneapolis 1986.

Coddou, A., Chadwick. (1994). M., Evolución del concepto de codependencia, Tesis. Universidad de Chile.

Delgado, D.,Pérez A., Análisis del fenómeno de la codependencia en familias de consumidores y no consumidores. Www.occsp.gov.co/downloads/invest/ codependencia. pdf.

Graña, J. (1988). Conductas adictivas: teoría, evaluación y tratamiento. Madrid, Editorial Debate.

Haaken, J. (1990). A Critical Análisis of the Codependence Concept Psychiatry, 53, 396 406.

Malpartida, C., Comportamiento del codependiente de pacientes con abuso de sustancias en 
cinco centros de tratamiento y rehabilitación de lima metropolitana. 1998. Tesis Universidad Peruana Cayetano Heredia, Lima, 1988.

Sirvent, C. (2,000). www.institutospiral.com/ symposium/resumen/sirvent.htm.

Vacca, R. (1998). Los padres los hijos y la pareja del adicto: de la familia funcional a la disfuncional. OPCION, Lima.

Withfield, C. (1991). Codependence, healing the human condition. Health Comunications. Inc., Florida. 